In Crescendo, 2019; 10(2): 387-407

Fecha de recepción: 28/02/2019

Fecha de aceptación: 06/06/2019

\title{
LA PARTICIPACIÓN PARENTAL EN EDUCACIÓN BÁSICA DESDE LA PERSPECTIVA DEL PROFESORADO*
}

\author{
THE PARENTAL INVOLVEMENT IN BASIC EDUCATION FROM THE \\ PERSPECTIVE OF THE TEACHING STAFF
}

\author{
Lorena Márquez-Ibarra ${ }^{1}$, María Luisa Madueño-Serrano ${ }^{1}$, \\ Alicia Carballo-Valenzuela ${ }^{1}$ Amairany Castillo-Barraza ${ }^{1}$
}

\section{RESUMEN}

El profesor se posiciona como una figura clave en la relación familia-escuela en los primeros niveles de educación. Se presentan los resultados de una investigación fenomenológica realizada en cuatro escuelas primarias de México cuyo propósito fue comprender la perspectiva del profesor sobre la participación de la familia en la educación. Participaron 17 profesores elegidos por muestreo selectivo y el tamaño de la muestra se determinó al alcanzar la saturación teórica. La técnica de recolección de datos fue la entrevista individual semiestructurada. El procesamiento de la información se realizó mediante análisis de contenido. Los resultados muestran que la participación de la familia en la educación de los hijos se reduce a informarse sobre su desempeño escolar y apoyar a la escuela con la organización de eventos y actividades para el mejoramiento de la infraestructura escolar. Surgen como barreras para la participación de los padres: la limitada disponibilidad de tiempo debido a los compromisos de trabajo, su bajo nivel económico y la poca importancia que se da a la educación de sus hijos. Se concluye que la participación es utilitaria y que existe desinformación sobre qué y cómo los padres pueden participar. Es necesario promover espacios que involucren a los padres en la educación de sus hijos en un ejercicio de reconocimiento mutuo y asociación para avanzar hacia el concepto de escuela como escenario de formación para la participación social, desde su estructura y organización.

PALABRAS CLAVE: Participación de la familia; educación básica; profesores; fenomenología.

* La presente investigación fue financiada con recursos del Programa de Fomento y Apoyo a Proyectos de Investigación (PROFAPI) y con recursos del Programa de Fortalecimiento de la Calidad Educativa (PFCE).

1 Instituto Tecnológico de Sonora. 


\begin{abstract}
The teacher is positioned as a key figure in the family-school relationship in the first levels of education. The results of a phenomenological investigation carried out in four primary schools in Mexico are presented, whose purpose was to understand the teacher's perspective on family participation in education. 17 professors participated chosen by selective sampling participated and the sample size was determined upon reaching theoretical saturation. The data collection technique was the individual semi-structured interview. The information processing was carried out through content analysis. The results show that the family's participation in the education of the children is reduced to being informed about their school performance and supporting the school with the organization of events and activities for the improvement of the school infrastructure. They arise as barriers to parental involvement: the limited availability of time due to work commitments, their low economic level and the little importance given to the education of their children. It is concluded that participation is utilitarian and that there is misinformation about what and how parents can participate. It is necessary to promote spaces that involve parents in the education of their children in an exercise of mutual recognition and association to advance towards the concept of school as a training scenario for social participation, from its structure and organization.
\end{abstract}

KEY WORDS: Parent participation; basic education; teacher; phenomenology.

\title{
INTRODUCCIÓN
}

La familia y la escuela constituyen dos de los principales pilares en la educación y socialización de las personas, por lo que la comunicación asertiva entre ambas es prioritaria para favorecer el desarrollo y aprendizaje de los educados. En la medida que el sistema escolar avanza, crecen en importancia las estrategias que se ponen en juego para establecer lazos de unión entre ambos entornos, esto debido a que, comunmente, en la etapa de preescolar, los padres y madres de familia se mantienen cercanos al centro escolar, pendientes de las necesidades escolares de sus hijos, y además, colaboran activamente con otros padres y los docentes; sin embargo, conforme los hijos crecen y avanzan de nivel educativo se ensancha la distancia entre la familia y la escuela (Márquez, 2014).

Por otro lado, es notable cómo la escuela va asumiendo roles que anteriomente eran conferidos a la familia, sobre todo en lo referente a socialización. Fernández (2016) señala que ese traslape está influído por las vertiginosas trasformaciones sociales que han rebasado los muros de ambos entornos, y además, se ha visto modificada la manera en que se asumen y valoran unos a otros (Andrés \& Giró, 2016). 
Por consiguiente, es imprescindible analizar la relación familia-escuela, en tanto que son los dos grandes agentes socializadores responsables de la educación de los niños y niñas, prestando atención a las realidades en las que están inmersos (Moratto, Zapata, \& Messager, 2015). Por su relevancia es necesario que familia y escuela trabajen conjunta y cooperativamente, por lo que ambos agentes requieren mantener vínculos constructivos y positivos, que sean copartícipes y corresponsables del proceso formativo de los alumnos; en esa medida, la participación de las familias favorece a la calidad de las escuelas y a la calidad de vida de las familias (Llevot \& Bernad, 2015; Belda, 2014).

La participación hace referencia a sentirse parte, ser parte y formar parte, pero también implica voluntad (Márquez, 2014). Para Llevot y Bernad (2015) participar es emprender acciones que afectan a un colectivo, las cuales van orientadas a la resolución de problemas que les son comunes. Para Márquez (2014), así como para Llevot y Bernad (2015), la participación de la familia en la escuela, en un sentido estricto, tiene que ver con correspondencia, involucramiento e intervenir en la toma de decisiones como miembro de la comunidad educativa para lograr un mismo fin. En la participación, además de voluntad se requiere saber cómo y en qué participar, pero también es necesario contar con las condiciones para hacerlo (Márquez, 2014).

Garreta, Llevot y Bernad (2011) y Garreta (2014) apuntan que, tanto para los docentes, representantes de las asociaciones de madres y padres de familia, asociaciones de alumnos y del personal directivo de las escuelas, la participación de las familias en la escuela y el que se mantenga una relación y comunicación fluida entre ambos, es clave para conseguir el éxito escolar del alumnado. En este sentido, la participación de la familia en la educación de los hijos puede ayudar a mejorar la calidad de los sistemas escolares, dado que, cuando esta se muestra participativa brinda un mosaico de oportunidades para que los hijos tengan éxito en su tránsito por la escuela (Collet, \& Tort, 2012; Machen, Wilson, \& Notar, 2005).

La participación de los padres en la educación de los hijos reviste una serie de beneficios tanto para ellos, como para los profesores y por consecuencia, para los estudiantes (Garreta, \& Llevot, 2015). Los padres desarrollan actitudes positivas, se muestran más satisfechos con los profesores, logran un mejor entendimiento del funcionamiento del centro escolar y sus programas, lo cual les mueve a participar más y asumir responsabilidades en la escuela. Por su parte los profesores ven facilitada su tarea y los alumnos alcanzan mejores resultados académicos; por 
consiguiente, el principal beneficiado es el estudiante y su desempeño escolar (Grant, \& Ray, 2013).

Por tanto, el profesor figura como un puente para la vinculación y comunicación con la familia y el centro escolar, principalmente en él recae mantener cercanas a las familias de sus estudiantes. Por ello, es importante continuar en el esfuerzo así se tengan infructuosos intentos de convocatoria (Trujillo, 2012). Es tal el impacto del profesor que pueden llegar a ser el principal agente promotor de la participación de los padres y madres de familia, pero también pueden actuar como un inhibidor (Comellas, 2009) y figurar como una barrera para la participación (Gomilla, \& Pascual, 2015) sin ser, quizá, conciente de ello.

Dado el importante papel que juega el profesor en el binomio familiaescuela, es que surgió el interés por desarrollar esta investigación y recuperar desde su propia voz y experiencia, la forma en que concibe esa relación y el rol que asume. Con lo anterior se espera adentrarse a una realidad educativa que de cuenta del entramado de prácticas y significados en torno a la participación parental, desde la mirada de uno de los actores educativos, que si bien se ha realizado en una comunidad específica, puede ser un reflejo de lo que pasa en otros entornos; en tal sentido, se espera que los hallazgos inviten a la reflexión y motiven a la acción para instrumentar estrategias que logren vincular a cada uno de los miembros que conforman una comunidad educativa para lograr los propósitos de formación que se persiguen.

\section{Participación parental en educación}

Los esfuerzos por afianzar el vínculo familia escuela siguen siendo una agenda pendiente, si bien, en la mayoría de los países se marcan directices precisas desde el marco legal educativo (Garreta, Llevot, \& Bernad, 2011), su concreción aún está endeble (Márquez, 2014). Afirman Perales y Escobedo (2016) que la participación de los padres en las escuelas es limitada y supeditada a las directrices que dispongan las autoridades educativas, señalan que si bien se habla de dar mayor autonomía a las escuelas, esta no aplica a todos los actores educativos.

Hablar de participación es hablar de relaciones de resistencia, que en cierta forma son naturales, toda vez que requieren un cambio en la cosmovisión no solo de los padres y madres de familia, sino de toda la comunidad educativa. El tema de la participación escolar necesariamente lleva implícito el surgimiento de cuestionamientos con respecto a relaciones de poder y responsabilidades. 
De acuerdo con (Anderson, 2002), una participación se considera efectiva cuando los sujetos de un grupo son capaces de construir intereses comunes y trabajar de forma voluntaria en torno a la problemática que los reúne, y de igual manera se deben seguir procesos organizativos con matices en los que se advierta igualdad, democracia y autonomía entre los miembros. De acuerdo con el autor antes citado, la participación, también debe ser auténtica, de tal manera que exista un involucramiento e intervención que tal forma que se vea reflejada en la toma de decisiones.

La participación de los padres en la educación de los hijos data de mucho tiempo atrás; sin embargo, su participación ha sido acorde a sus expecttivas, intereses o necesidades, así como de los límites que imponen los centros educativos y el marco legal de la educción vigente (Perales, \& Escobedo, 2016). Visto como un proceso complejo, la participación familiar en las escuelas involucra diferentes ámbitos y niveles de acción, requiere desde brindar apoyo indirecto al aprendizaje de los hijos en casa, hasta colaborar con la escuela e intervenir en la toma de decisiones, esto en coordinación con otros actores educativos (Epstein, \& Sheldon, 2007).

La participación parental incluye las diferentes actividades que padres y madres o tutores realizan tanto con los hijos, la escuela y la comunidad, lo anterior con el propósito de mejorar el logro académico de los estudiantes (Lines, Milles, \& Arthur-Stanley, 2011). La participación parental puede ser individual o colectiva. Los padres participan individualmente asistiendo a reuniones, participando en las actividades que organiza la escuela, en el seguimiento escolar de sus hijos, entre otras; colectivamente, al organizarse con otros padres y madres de familia de manera formal en asociaciones, comités o formando parte de los consejos escolares.

Epstein y Sheldon (2007) sostienen que la participación permite desarrollar interacciones efectivas que facilitan una mejor comprensión de los diversos puntos de vista; la formulación de metas comunes para los estudiantes; asimismo, la comprensión de los esfuerzos mutuos a partir del rol de cada uno de los actores del proceso educativo, y el intercambio oportuno de información entre la comunidad escolar.

Además de lo anterior, otra de las ventajas del involucramiento de los padres y madres de familia en la escuela es el aumento de las oportunidades de aprendizaje del estudiante (Valdés, Martín, \& Sánchez, 2009). Desafortu- 
nadamente el asunto se complejiza porque queda a voluntad de los progenitores o tutores de los alumnos esta decisión, de tal manera que pocos se comprometen a intervenir de forma directa en la escuela y en la educación de sus hijos; o bien, su margen para hacerlo es limitado desde el propio centro escolar (Marquez, 2014).

La idoneidad de la participación de los padres, en ocasiones es limitada debido a su situación económica y laboral, en muchas ocasiones se requiere que tanto el padre como la madre de familia salgan a trabajar, y eso dificulta las labores familiares y el brindar apoyo en casa en las tareas escolares de los hijos, pudiendo verse afectado su rendimiento escolar.

Por otra parte, Valdés, Martín y Sánchez (2009) señalan que el nivel de estudios de los padres se correlaciona de manera positiva con su participación pero al mismo tiempo se encuentra que esta es baja; en ese sentido, Díaz, Pérez y Mozó (2009) encontraron que el nivel de escolaridad y expectativas de padres y madres es una de las variables que influye en el rendimiento de los hijos y se manifiesta principalmente en comportamientos de apoyo hacia el estudio.

La participación de los diferentes agentes de la comunidad educativa desempeña un papel decisivo para garantizar el éxito académico, social y emocional de un niño (Lines et al., 2011). Este escrito se centra principalmente en dos agentes, por un lado los profesores, y por el otro los padres y madres de familia o tutores; esta relación requiere del trabajo conjunto y coordinado entre ambas figuras (Belda, 2014; Comellas, 2009; Fernández, 2008; Epstein, 2001) para incidir en el desarrollo educativo de los niños y adolescentes (Collet \& Tort, 2012).

Para generar condiciones que favorezcan la participación de los padres en la vida escolar, es ingrediente necesario que los profesores estén conscientes del valor que posee el involucramiento de estos en su tarea educativa y de cómo el hacer sinergia potencializa el logro de los objetivos de aprendizaje. Pero esta consideración debe ser bidireccional, toda vez que ambas figuras reconozcan y valoren la aportación y potencial del otro. Para ello es imprescindible generar y mantener canales adecuados de comunicación y facilitar momentos y oportunidades que favorezcan la relación y convivencia (Ellis \& Hughes, 2002). Es por lo anterior que esta investigación tuvo como objetivo comprender la perspectiva del profesor de educación básica sobre la participación de la familia en la educación de sus hijos. 


\section{MÉTODO}

La presente es una investigación cualitativa fenomenológica que se interesa por la evidencia vivida (Reeder, 2011); se orienta al abordaje de la realidad partiendo del marco de referencia interno del individuo, que en este caso es el docente. En el ámbito de la educación, la fenomenología contribuye a dar cuenta de la realidad en las escuelas especialmente a recuperar las vivencias de los diversos actores educativos (Aguirre \& Jaramillo, 2012).

De acuerdo con Montero (2007) y Álvarez (2011) el método fenomenológico permite el abordaje de la subjetividad del individuo, sus sentimientos, recuerdos, vivencias que el investigador pone de manifiesto tratando de comprender cómo este construye significados y lo logra mediante la descripción de los significados vividos, existenciales, aquíla experiencia es el foco de estudio.

Se expone a continuación el referente empírico donde se aborda el escenario de estudio, criterios de selección de los informantes y tamaño de la muestra así como las consideraciones éticas y criterios de rigor atendidos. El segundo apartado detalla la técnica empleada para recuperar información y el procedimiento de análisis.

\section{Referente empírico}

Para la elección de las escuelas participantes en este estudio se consideraron tres aspectos: el primero que sean de sostenimiento público para indagar la perspectiva de los docentes con padres con diferentes niveles socioeconómicos, el segundo punto fue que se ubicaran en diferentes zonas de la ciudad, que fueran escuelas de tiempo completo y se contara con la autorización expresa de los directivos para su realización.

Participaron un total de cuatro instituciones de educación primaria, que va desde los 6 a los 12 años, situadas en cuatro colonias de nivel socioeconómico medio bajo de una comunidad urbana del norte de México. Con fines de resguardar el anonimato tanto de los centros educativos como de los informantes se utilizó homoclaves con fines de diferenciación, ésta estuvo conformada por la letra E, seguido de un número que hacen referencia a la escuela (va de E1 a E4), seguido de la letra P y un número consecutivo que hace referencia a los participantes (P1 a P15).

Los participantes en el estudio fueron profesores de primero a sexto grado, se eligieron mediante muestreo selectivo (Martínez, 2012; Naupas, Mejía, Novoa, 
\& Villagómez, 2014). Los criterios fueron: contar con mínimo cinco años de experiencia impartiendo clases en ese centro educativo y que aceptaran voluntariamente participar en la investigación. Se cuidó la equidad de género dado que fueron ocho profesoras y siete profesores los elegidos cuya edad promedio fue 41 años.

Para la realización de las entrevistas se tomó en cuenta las consideraciones éticas exigidas en estudio de corte cualitativo. Se contó con el consentimiento informado de los participantes del estudio para garantizar su autonomía y respeto en todo momento. Para asegurar los criterios de rigor metodológico, la confidencialidad se atendió respetando el anonimato al reemplazar datos de identificación por homoclaves en una combinación de escuelas y participantes. La coherencia metodológica se atendió mediante la congruencia entre la pregunta de investigación, método, técnica de recolección de información y método de análisis (Salgado, 2007); y la credibilidad se logró al transcribir fielmente las entrevistas en las que se recuperó la narrativa tal como fue expresada por el informante.

El tamaño de la muestra se determinó mediante el criterio de saturación teórica, razón por la cual se consideraron hasta cuatro escuelas. La saturación teórica es un criterio que pretende la riqueza explicativa, busca recopilar datos hasta saturar todas las categorías para que se desarrolle la teoría equilibradamente. La saturación permitió identificar, mediante comparaciones constantes, el momento en que fue necesario detener la recolección de datos porque estos dejaron de aportar información nueva y valiosa (Corbin \& Strauss, 2014; Flick, 2007).

\section{Recolección de evidencias}

Para la identificación de las escuelas primarias se contó con un listado del que se fue seleccionando las que se visitarían según los criterios antes mencionados. Se realizó contacto vía telefónica para concertar cita con el directivo escolar con la intención de entregar un oficio que detalló los fines, método y alcances de la investigación, asi como los compromisos que asumiría el cuerpo de investigadores. Durante esa reunión se atendieron dudas e inquietudes y se solicitó autorización para realizar el estudio.

Posteriormente se procedió a identificar y contactar a los profesores de acuerdo con los criterios de selección preestablecidos y se les explicó el propósito del estudio, el manejo confidencial y anónimo de la información que aporten, el 
uso que se haría de la misma, así como el requerimiento de grabar en audio la entrevista, para lo cual expresaron y firmaron su consentimiento.

Las entrevistas fueron semiestructuradas e individuales y se realizaron en un espacio dentro de las instalaciones de las escuelas con la privacidad suficiente para evitar interrupciones y favorecer el dialogo. Estas se apoyaron en una guía de entrevista que contenía tópicos y preguntas detonantes que facilitaron guiar la conversación en torno al fenómeno en estudio, su grado de estructuración otorgó libertad para ajustar la exploración de los temas.

Los audios de las entrevistas fueron transcritos en el procesador de textos word y para su procesamiento se realizó un análisis de contenido (Martínez, 1988; Bolívar, 2012) que consistió en un procedimiento de categorización, análisis e interpretación. La transcripción fiel de las narrativas fueron revisadas en varias ocasiones hasta captar las ideas, palabras relevantes y significativas de acuerdo con los propósitos del estudio, y posteriormente se dividió el contenido en unidades temáticas o de significación, que de acuerdo con Márquez (2014) representan núcleos duros de sentido; es decir, son párrafos que expresan una idea o concepto central.

El procedimiento de categorización se realizó al momento de agrupar la información mediante la expresión del contenido de cada unidad temática que son tópicos a través de los cuales se organiza la información (Cisterna, 2005). Esta fase es un proceso cognitivo para organizar información obtenida de la percepción de la realidad, que consiste en extraer de un texto segmentos de contenido o dimensiones (Martínez, 1988). Dicho momento se realizó tomando en cuenta las evidencias obtenidas y según la frecuencia y relevancia de cada unidad de significación se fueron identificando las categorías que englobaban lo relacionado con esa temática.

Posteriormente, se analizó la información mediante el método de triangulación, en este caso, de fuentes. Se optó por la triangulación por la posibilidad que ofrece de identificar patrones de convergencia que ayuden en la interpretación global del fenómeno en estudio, pero además por su contribución a la rigurosidad metodológica en la investigación y porque favorece análisis exhaustivos y más reflexivos (Mays \& Pope, 2000; Patton, 2002). Este consistió en registrar las expresiones de los participantes por cada categoría, las cuales fueron consideradas como evidencias, las fuentes de información teórica relacionada a la categoría y finalmente se realizó la interpretación con lo cual los datos cobraron sentido. 
Por último se pasó a la discusión de resultados en un entramado conformado por evidencias, cuerpo teórico e interpretación que facilitó hacer inferencias respecto al objeto de estudio y derivar las conclusiones.

\section{RESULTADOS}

Como en cualquier proceso educativo, la participación parental en las escuelas involucra una serie de aspectos, tanto normativos, cultura escolar, condiciones sociodemográficas, curriculares, posturas e interpretaciones diversas entre los actores educativos, todo ello va preconfigurando un esquema de valores, creencias y formas de accionar, es decir se construyen y reconstruyen significados, no en forma aislada sino a través de la experiencia y de las interacciones con otros. Lo anterior son construcciones sociales, pero no por ello similares, por lo que ahí surge el interés de explorar las miradas y su diversidad ante un mismo fenómeno educativo.

A partir de los hallazgos de la presente investigación, se identificaron cuatro categorías emergentes, las cuales se conformaron a través del análisis temático de la información: a) significados sobre la participación parental, b) prácticas de participación, c) barreras para la participación, d) entre lo real y lo ideal. Cada una de ellas serán abordadas en los apartados siguientes.

\section{Significados sobre la participación parental}

La pertinencia de incorporar en este estudio la voz del docente provee pautas para profundizar en el fenómeno de estudio, a través de conocer cómo viven y conciben la participación de los padres y madres de familia en la educación. En las narrativas de los profesores participantes surgieron indicios sobre lo que para ellos significa la participación de padres y se encontraron coincidencias y divergencias entre las respuestas de los participantes.

Hay quienes asumen la participación con involucrarse en las actividades educativas de sus hijos y de la escuela, así lo expresan: "Que los papas se involucren en las actividades propuestas por la escuela y también en la educación de los niños" (E4P14), "La manera en la que se involucran en el aprendizaje de sus hijos y la escuela, y no nomás en los aprendizajes, si no en las mejoras de la escuela" (E4P12), "Que se involucren con los niños, que los apoyen, que los manden a la escuela, que les den alimento, que estén al pendiente de ellos, colaborar, ayudar al niño, estar al pendiente, dar un seguimiento" (E1P2) y otro participante lo concibe como "El grado en que se 
involucran los padres de familia ya sea en el aprendizaje de sus hijos o cualquier otro aspecto que se pueda presentar [...] incluirte en las actividades que se realizan" (E1P3).

En su discurso se destaca que tal involucramiento lo refieren a lo concerniente al aprendizaje de sus hijos, y también hacen mención a implicarse para hacer mejoras en las escuelas. Otros padres de familia se extienden más allá de estas concepciones y asumen que la participación de los padres es estar pendiente, dar apoyo a los hijos y colaborar con la escuela y con el resto de los actores educativos. Se destaca en sus discursos una coincidencia con el planeamiento de Márquez (2014) quien precisa que participar implica estar, formar parte y sentirse parte de esa comunidad, lo anterior con miras a favorecer la educación de sus hijos, así lo mencionan tres de los entrevistados: "Es apoyar, estar siempre al pendiente y conocer las necesidades de la escuela, para así ser partícipe de ella" (E3P10), "Que estén al pendiente y apoyen a sus hijos en las actividades extra escolares [...] realizar las actividades en equipo, padres de familia, maestros, alumnos, directivos, que cada quien haga su función en lo que le corresponde" (E3P9), y "Que los padres colaboren en las actividades que se realizan, que aporten, pregunten, se involucren; es ayudar, aportar" (E4P13).

Señalan los profesores que no solo es brindar apoyo al hijo, sino que también es asegurarse que vaya a la escuela, que tenga lo necesario para poder adquirir los aprendizajes esperados, así como estar al pendiente de sus necesidades y las de la escuela, además de apoyarlos con sus tareas y proyectos. Se deja ver en sus narrativas que la representación que configuran sobre la participación de los padres y madres lleva incrustada la necesidad de que su labor docente se vea beneficiada al tener su colaboración.

A partir de los significados sobre la participación parental, se identifican atributos relacionados con una concepción que destaca el emprender acciones para atender o solucionar problemas cuya finalidad última sea el beneficio para los hijos; y de igual manera, cobra presencia la necesidad de que los padres formen parte y esten para sus hijos desde su rol como agentes de la comunidad escolar, además de sentirse parte de ella.

\section{Prácticas de participación}

En el contexto escolar se presenta un entramado de relaciones, actores, roles, expectativas, creencias y prácticas, tejido que es matizado en función de las normativas educativas vigentes, el currículum y la cultura escolar. La actua- 
ción de los padres y madres de familia puede darse tanto dentro como fuera de la escuela, en este sentido, puede manifestarse en una diversidad de formas y grados, pero también entenderse y apreciarse de manera diferente por el resto de los actores educativos. Señala Gómez (2011) que en la medida en que los individuos configuran sus representaciones, es la forma en que actuan ante ellas.

Las representaciones que sobre la participación de los padres poseen los profesores dan cuenta sobre su forma de percibir, entender y accionar ante esa participación. Cabe mencionar que en ese proceso de configuración, que lleva a la actuación, la experiencia de vida juega un papel importante (Cuadra, 2008; Arbesú, Gutiérrez y Piña, 2009), por tanto, en este apartado se retoma lo expresado por los entrevistados en torno a las prácticas que realizan los padres y madres de familia, también da cuenta de las referidas al propio profesor y su rol en la relación familia-escuela, así mismo, se trastocan las prácticas que se promueven desde la gestión escolar.

A decir de los profesores entrevistados, algunos padres participan más que otros, señalan que existen padres que llevan a cabo actividades de involucramiento al trasladar a sus hijos al centro escolar, proveerles los materiales que requieren y brindarles apoyo con sus labores escolares desde casa o al supervisarlas; en cambio hay otros que llegan a tener mayor presencia dentro del espacio escolar asistiendo a las reuniones convocadas, a talleres para padres, agrupándose con otros padres y madres de familia para conformar asociaciones de padres y comités, cuyas funciones y personalidad es reconocida oficialmente por la escuela.

Otras vías de participación es la colaboración con la escuela en la organización de actividades o eventos, ya sean estos ceremoniales o actividades recreativas (fin de cursos, graduaciones, festejos del día del maestro, de las madres, del niño, excursiones, eventos deportivos, etc) o para la recaudación de fondos (kermes, rifas, vendimias), estas últimas con el propósito de reunir recursos económicos para realizar mejoras estructurales o adquirir mobiliario para la escuela; así lo expresa una profesora:

[los padres participan] cuando le pedimos apoyo para un festejo, apoyo para pintar la escuela, vienen y ayudan, así participan, ayudan a cuidar los planteles, las instalaciones, cuando hay algún torneo deportivo, alguna fiesta escolar, alguna kermes, es cuando se les llama para entrega de calificaciones (E1P4). 
El anterior extracto de entrevista destaca coincidencias con lo expresado por cinco participantes más (E2P8; E2P5; E3P9; E3P10; E4P13) en cuanto a colaborar con la escuela. Esta colaboración es respaldada y promovida desde los marcos legales educativos, con lo cual se busca sean copartícipes del proceso educativo de sus hijos (Llevot y Bernad, 2015).

En cuanto a los espacios que desde la escuela se favorecen para involucrar a los padres, los entrevistados expresan que son invitados o que pueden estos involucrarse por voluntad en actividades o eventos que realiza la escuela. Recalcan que desde el principio del ciclo escolar se les comunica la manera de trabajar, y les hace saber sus responsabilidades y obligaciones dentro y fuera de la escuela.

Por su parte los profesores llevan a cabo acciones para reconocer a los que más participan mediante la entrega de distintivos o premios para motivar la participación parental: "muchas veces les mandamos a hablar... que vamos a tener entrega de calificaciones, que le vamos a dar un punto al padre de familia que asista" (E1P4); lo anterior, además de otras acciones dirigidas a sensibilizar sobre la importancia de su participación en el desempeño escolar de sus hijos, así lo narra una profesora: "hacerles ver que la participación de ellos no es nomas por requisito [...] porque si ellos están, le ayuda al niño y a mí también como maestra" (E4P14) y en ese intento de que reconozcan el valor de su participación hay quienes hacen alusión a apartados específicos de la Ley General de Educación de México:

Yo platico con los padres y les hablo claro: "En el artículo $3^{\circ}$ Constitucional y en la misma Constitución están los derechos de los niños y las obligaciones de los padres, aunque uno no sepa, violentamos los derechos de los niños". Uno como padre violenta los derechos, al descuidarlos (E2P7).

Y agrega (E2P7) que ellos, como padres, tienen libertad de reportar ante las autoridades las irregularidades que observen en los profesores y deja entrever que también es necesario que ellos se mantengan cercanos a sus hijos y la escuela.

El profesor es también el encargadado de invitar a los padres y madres de familia a reuniones para tratar asuntos relacionados con sus hijos. Los profesores participantes, mencionan que esas reuniones son para entrega de calificaciones bimestrales, para organizar algún evento o para atender situaciones específicas en las que se cita en lo individual a los padres. También se hace mención a la apertura de recibir a los padres cuando tienen una inquietud particular, así lo expresó un profesor: "ellos tienen la puerta abierta a mi salón, exclusivamente a mi salón, ellos 
pueden venir cuando ellos quieran y a la hora que quieran, yo tengo las puertas abiertas para ellos siempre" (E3P9).

En su discurso los entrevistados coincidieron en que el medio de comunicación con los padres son las notificaciones por escrito (recados, citatorios o avisos) las mismas que se hacen llegar a través los estudiantes.

En síntesis, las principales prácticas que llevan a cabo los padres de familia, desde la perspectiva de los profesores, se aprecian en dos formas. Por una parte, participaciones en las que los padres actúan como provedores y desde las cuales se aseguran de que sus hijos asistan a la escuela, ejemplo de ello es comprarles material requerido y llevarlos a la escuela; este tipo acciones, de acuerdo con Perales y Escobedo (2016) se considera limitada y escasa.

Por otra parte, se advirtieron prácticas de mayor involucramiento en diversos ámbitos y niveles de acción, como asistir a la escuela y conformar grupos de apoyo para la escuela y para sus hijos. Asimismo prevalecen prácticas como asistir cuando se les convoca por los docentes o directivos, participar en las actividades organizadas por la institución, colaborar para que existan mejores condiciones e infraestructura en la escuela para el beneficio de sus hijos; esto último, como lo plantean Epstein y Sheldon (2007) refiere a prácticas más próximas a una participación informada y con mayor grado de involucramento.

\section{Barreras para la participación}

Otra de las categorias emergentes desde el análisis de los datos hace referencia a las barreras para que los padres y madres de familia participen en la educación de sus hijos desde la mirada del profesor. Participar hace pensar en pertenencia, en apropiación, en cooperación y corresponsabilidad. No es un concepto que se conjuga en lo individual, sino que involucra a otros. Requere, de quien participa, voluntad para hacerlo, conocimientos para determinar cómo y en qué participar, pero también es preciso que exista oportunidad para hacerlo; es decir que se den las condiciones propicias (Márquez, 2014), y en este caso, corresponde a las escuelas el que se favorezcan espacios y momentos para involucrar, en el compromiso educativo, a todos los actores, entre ellos, los padres.

Tener la voluntad para participar implica tener deseos de hacerlo, valorarlo como importante o necesario, requere motivación para luego movilizarse en torno a eso que se desea. En este sentido, desde los discursos de los entrevistados se deja ver que en ocasiones, el profesor es quien lleva a cabo determina- 
das estrategias para motivar a los padres que denotan apatía de su parte. A este respecto uno de los profesores comentó que es necesario "estar estimulándolos, estar diciéndoles la importancia de participar, una manera por decir... es tomando en cuenta su participación para una materia de sus hijos. Sí participan se le suben puntos" (E3P11). Por otro lado se resaltó el trabajo de los padres como un obstáculo, un profesor expresó: "deben trabajar, o no se dan tiempo para acudir y ponerse en contacto con nosotros, los padres ahora trabajan y sus horarios de trabajo y los de la escuela son los mismos y no tienen tiempo para hacerlo" (E4P13). Y bajo esa misma tónica otro señaló:

quiero pensar yo que es por cuestiones laborales, que quizá tienen el mismo horario de trabajo que nosotros, a lo mejor aquel padre de familia que no asiste a una reunión, que no asiste al llamado del maestro puede ser porque trabaja a esas horas y nole permite su horario asistir (E4P15).

El trabajo de los padres es una cuestión recurrente en las investigaciones realizadas sobre este tema, pero tambien viene asociado con poca importancia a la educación de los hijos o a un bajo interés. A partir de los resultados de la presente investigación, restar interés e importancia a participar, es una barrera, así lo expresó uno de los entrevsitados: "siempre ponen el pretexto del trabajo, que no tienen tiempo, que tienen más hijos, que tienen que trabajar para mantenerlos - el único factor que ponen es su trabajo y la falta de tiempo, no tienen interés" (E3P11), en ese mismo tono una profesora narra:

muchos se justifican "es que mi trabajo" yo siento que es eso, como en este caso que es primero, "va empezando, no ocupa mi apoyo" o "es que no tengo tiempo, por el trabajo" o a veces por la vida acelerada que llevamos me dicen "sí, mañana, mañana" y no vienen, o dicen "mi hijo no es problemático, va bien en la escuela, no ocupo ir" (E2P5).

Señala Márquez (2014) que los padres participativos irrumpen y rompen barreras, dado que, lo que los mueve es la aspiración a una mejor educación para sus hijos y reconocen su propio potencial para lograrlo, además de que buscan convencer a otros padres y madres para ganar mayor fuerza en sus propósitos.

Otra de las barreras que surgen a partir de los discursos de los participantes es el bajo nivel económico de los padres, ellos expresan: "lo que pasa es que los 
padres de familia, la mayoría en esta escuela, son de bajos recursos y tanto papá como mamá tienen que trabajar, entonces por eso tienen en el descuido a los niños" (E3P9). Otro de los entrevistados coincide:

Es la economía, ahora papá y mamá tienen que trabajar y no pueden estar al pendiente de lo que el niño requiere. No pueden asistir a reuniones por que trabajan, asistir a una reunión significa que les van a descontar de su trabajo (E4P12).

Además de lo anterior, hacen señalamientos con respecto a otros factores sociodemográficos: "tenemos muchos problemas de familias disfuncionales, padres solteros, madres solteras, padres adictos a una droga, son de extrema pobreza, la mayoría trabaja, entonces todo eso influye en los niños, están muy mal atendidos ahorita los niños" (E3P9). Otro narra: "hay muchas familias disfuncionales, y la drogadicción que, no nomás está en los jóvenes, también esta en muchos padres de familia, el alcoholismo que se ve mucho en la sociedad y que ya rebaso los limites" (E4P12). O bien, el desconocimiento:

muchas veces piensan que es estar todo el tiempo aqui en la escuela "es que tengo que ir todo el día a la escuela" "es perder tiempo, por ir a reuniones, actividades" muchas veces dicen "es que tengo que trabajar" [cuando se les pide su apoyo] (E2P5).

Las principales barreras tienen un origen de tipo socio-económico asociado con la necesidad de laborar de ambos padres, aunque también se identifica que la falta de voluntad por parte de ellos se hace presente al escudarse en la falta de tiempo y en esos casos, de acuerdo a lo planeado por los profesores, la incompatibilidad de horario, para participar, es una justiicación recurrente por parte de los padres.

\section{Entre lo real y lo ideal}

Para los profesores el ideal en la participación con respecto a los padres, sería que esta fuera activa, estando al pendiente de ellos, tanto en los aspectos que pudieran tratarse dentro de la escuela como puede ser la conducta, calificaciones, como también apoyando a los niños en la elaboración de tareas, cumplir con lo que se les solicita. Así lo expresan: "el padre de familia debe apoyar al niño en sus quehaceres académicos como tareas, trabajos individuales y en equipo [...] es su responsabilidad el cumplimiento de las actividades educativas de su hijo" (E2P8), "el padre de familia debe de estar al pendiente de la evolución tanto académica como personal del 
alumno [...] que atiendan las indicaciones que el docente les $d a$, porque obviamente son por el bien de su hijo" (E4P15); "deben estar al pendiente de sus hijos en las tareas escolares, en las actividades que hace la dirección, en los festivales, apoyar a los hijos, estar al pendiente de las calificaciones, de sus trabajos, sus cuadernos, sus libros" (E3P9).

Además, los profesores entrevistados esperan que los padres y madres de familia asistan a la escuela cuando sea necesario, apoyen al profesor, mantengan comunicación, expresen su sentir, inconformidades o sugerencias, pidan apoyo cuando lo requieran. También mencionan que les gustaría que apoyaran con el mantenimiento de la escuela, ayudando con la limpieza, vigilancia en tiempos vacacionales, siempre y cuando ellos puedan hacerlo. Así lo señalan: "el padre de familia tiene que tener una participación importante en la escuela, en todos los ámbitos: educativo, organización, económico" (E2P7).

Sin embargo, la realidad sobre participación en las escuelas es que muy pocos padres participan, $\mathrm{y}$ lo hacen asistiendo solamente a las reuniones que se realizan de manera bimestral y no todos: "en las juntas cuando se van a dar las calificaciones, asisten muy pocos padres osea como que no se preocupan realmente como le esta yendo a su niñoniña porque hay muy poca participación de ellos en las juntas" (E1P3).

Los profesores mencionan que la participación no es la deseable, pocos atienden los llamados que se hacen para las reuniones y además, llama la atención el que se sientan fiscalizados o juzgados por los padres: "a veces pareciera que lo entienden como si su participación es más fiscalizadora hacia nosotros, como que son jueces de nuestro trabajo" (E4P15). En esa misma línea, otro participante del estudio expresa:

muchas veces los papás se toman atribuciones que no les corresponde o simplemente realizan comentarios inadecuados [...] sin bases y levantan una cortina de humo, la cual no deja ver la labor que hace el docente y muchos padres de familia llegan echando la culpa sin saber (E2P8).

Los padres envían a otras personas a los centros escolares en su representación, señalan los profesores que se ve a los centros educativos como una especie de guardería donde pueden dejar a sus hijos mientras ellos se encuentran trabajando o realizando alguna otra actividad: "creo que ellos piensan que con solo venir a dejar a sus hijos, y asistir por las calificaciones es suficiente" (E4P13).

Se tuvo la oportunidad de recuperar experiencias que los participantes consideran positivas o gratificantes con respecto a los padres de sus estudiantes a lo 
largo de su práctica docente, gran parte de ellos mencionan que la mejor experiencia que pueden tener es cuando se sienten apoyados por los padres de familia, cuando asisten y participan de manera activa en las actividades requeridas, cuando observan mejorías en sus alumnos, ya sea académica, conductual o con relación a otro aspecto en el cual presentaban dificultad. Una profesora compartió una anécdota:

en mi primer año de servicio me tocó un grupo en el que se me advirtió: "los padres de familia no participan ni para bien, ni para mal, vas a hacer las juntas y no vas a tener más que dos o tres papás, no vienen casi, el grupo también esta demasiado inquieto", incluso me dijeron con otras palabras. Pero a pesar de todo eso, logré que se integraran más los padres de familia, en el sentido que yo pienso que se deben de integrar. A la mayoría de los alumnos se logro inchir en las modalidades de trabajo que se implementaron, nunca se logra el trabajo al 100\% pero el $90 \%$ sí logré el avance que yo deseaba (E4P15).

En el plano de lo ideal, los profesores esperan una participación activa por parte de los padres, desean sentirse apoyados desde las actividades que ellos proponen, y en ocasiones, además de encontrarse con una realidad opuesta a ese ideal, se llega a sentir un dejo de crítica hacia ellos.

\section{DISCUSIÓN}

En el proceso educativo formal de niños y jóvenes la participación parental es un elemento clave, en tanto se formen alianzas positivas y constructivas entre ellos y en centro escolar. En este sentido, la comunicación y colaboración permiten formar un frente común para lograr el éxito escolar de los estudiantes.

Se ha hecho notar a lo largo de este escrito que la participación en sí misma amerita llevar a cabo acciones y tomar decisiones en conjunto, pero también, asumir responsabilidades. En las escuelas son varios los actores que le dan sentido: las autoridades educativas, personal administrativo, docente, estudiantes, los padres y la comunidad; si bien es conveniente recuperar sus voces, en la presente investigación se hizo un acercamiento solamente a los docentes, en un intento de comprender su perspectiva sobre la participación de los padres en la educación y su propia participación en la relación familia-escuela. La ruta trazada deja inquietudes que habrán de atenderse para llegar a profundizar en el fenómeno de la participación de los padres desde otra mirada, en la que se conjugue los imagina- 
rios que se gestan dentro y fuera de los muros escolares y que permean las prácticas y representaciones de quienes hacen posible el hecho educativo, asimismo, analizarlo desde la lupa de la gestión escolar y su marco normativo.

De acuerdo con los hallazgos, la voluntad de los padres para participar muestra matices en los que en ocasiones se ve desdibujada dicha voluntad y por otro lado, se reconoce cómo otros padres sí muestran interés para involucrarse y para estar informados respecto a cómo apoyar a partir de las necesidades de la escuela y de sus hijos; a este respecto se encuentra una coincidencia con el planeamiento de Márquez (2014) quien señala que una condición necesaria para favorecer la participación es que los padres identifiquen cómo y en qué participar.

Por último, es importante destacar que las expectativas que tiene el profesor con relación a la participación de los padres están matizadas con lo que ellos consideran como un deber: el trabajo conjunto entre la familia y la escuela para beneficiar a los alumnos; sin embargo, la realidad es otra, los hallazgos dejan una agenda pendiente por atender desde la gestión escolar para generar o bien, fortalecer condiciones que propicien el involucramiento y participación de la familia en la escuela. Es necesario seguir examinando desde diversos ángulos el fenómeno de la participación participación social en la escuelas dado que, en la medida que se logre una mayor comprensión se podrán definir rumbos de acción efectivos y con ello transitar hacia una cultura de participación y por ende una mejora de la educación.

\section{REFERENCIAS BIBLIOGRÁFICAS}

Aguirre, J. C., \& Jaramillo, L. G. (2012). Aportes del método fenomenológico a la investigación educativa. Revista Latinoamericana de Estudios Educativos, 2(8), 51-74.

Álvarez, E. (2011). La cuestión del sujeto en la fenomenología de Husserl. Investigaciones fenomenológicas, 8, 101-158.

Anderson, G. L. (2002). Hacia una Participación Auténtica: Deconstrucción de los Discursos de las Reformas Participativas en Educación. En M. Narodowski., M. Nores., \& M. Andrada (eds). Nuevas tendencias en Políticas Educativas. Buenos Aires: Temas/fundación Gobierno y Sociedad (pp. 1-61). Buenos Aires: Temas Fundación Gobierno y Sociedad.

Andrés, S., y Giró, J. (2016). La participación de las familias en la escuela: una cuestión compleja. Revista de Evaluación de Programas y Políticas Públicas, 7, 28-47. doi: 10.5944/reppp. 7.2016.16302

Arbesú, M., Gutiérrez, S., y Piña, J. (2008). Representaciones sociales de los profesores de la UAM$\mathrm{X}$ sobre la evaluación de la docencia e investigación. REencuentro. Análisis de Problemas Universitarios, 53, 85-96. 
Belda, J. F. (2014). La participación social en la escuela: un reto colectivo. Anthropos, 283, 83- 102.

Bolívar, A. (2012). Metodología de la investigación biográfico-narrativa: recogida y análisis de datos (Dimensões epistemológicas e metodológicas da pesquisa auto-biográfica). Porto Alegre, Portugal: Editora da PUCRS

Cisterna, F. (2005). Categorización y triangulación como procesos de validación del conocimiento en investigación cualitativa. Theoria, 14(1), 61-71

Collet, J., \& Tort, A. (2012). Famílies, escola $i$ èxit. Milloar els vincles per millorar els resultats. Barcelona: Fundació Jaume Bofill.

Comellas, M. J. (2009). Familia y escuela. Compartir la educación. Barcelona: Graó. Colección Familia y Escuela.

Corbin, J., \& Strauss, A. (2014). Basics of Qualitative Research: Techniques and Procedures for Developing Grounded Theory. United States of America: SAGE.

Cuadra, D. J. (2008). Representaciones sociales de niño integrado y proyecto de integración escolar en escuelas básicas con y sin integración. REICE. Revista Electrónica Iberoamericana sobre Calidad, Eficacia y Cambio en Educación, 6(3), 121-138.

Díaz, A., Pérez, M., y Mozó, P. (2009). Expectativas educacionales hacia hijas e hijos en una escuela rural de alto desempeño. Revista Interamericana de Psicología, 43(3), 442-448. Recuperado de http://www.psicorip.org/Resumos/PerP/RIP/RIP041a5/RIP04347.pdf

Ellis, D. \& Hughes, K. (2002). Partnerships by Design: Cultivating Effective and Meaningful SchoolFamily-Community Partnerships. Portland: Northwest Regional Educational Laboratory.

Epstein, J. L. (2001). School, Family and Community Partnerships. Preparing Educators and Improving Schools. Boulder: Westview

Epstein, J. L., \& Sheldon, S. (2007). Moving Forward: Ideas for Research on School, Family, and Community Partnerships. In C. F. Conrad., \& R. C. Serlin (Eds.). Handbook for research in education: Engaging ideas and enriching inquiry (pp. 117-137). Thousand Oaks, CA: Sage Publications.

Fernández, M. (2008). Educar es cosa de todos: escuela, familia y comunidad. En J. Garreta (Ed.), La relación familia-escuela (pp. 13-32). Lleida, Ediciones Universitat de Lleida.

Fernández, M. (2016). La Educación en la encrucijada. Madrid: Fundación Santillana.

Flick, U. (2007). Introducción a la investigación cualitativa. Madrid: Sage.

Garreta, J. (2014). La participación de las familias en la escuela: una cuestión pendiente. Documentación Social. Revista de Estudios Sociales y Sociología Aplicada, 171, 101-124.

Garreta, J. (2015). La comunicación familia-escuela en Educación Infantil y Primaria. Revista de la Asociación de Sociología de la Educación, 8 (1), 71-86.

Garreta, J., \& Llevot, N. (2015). Family-school communication in Spain: channels and their use. Ehquidad International Welfare Policies and Social Work Journal, 3, 29-48. doi: 10.15257/ehquidad.2015.0002

Garreta, J., Llevot, N., \& Bernad, O. (2011). La relació família d'origen immigrat i escola primària de Catalunya. Barcelona: Fundació Jaume Bofill.

Gómez, E. N. (2011). Habitar el lugar imaginado. Formas de construir la ciudad desde un proyecto educativo. Guadalajara, México: ITESO 
Gomilla, M. A. y Pascual, B. (2015). La participación de las familias en el sistema educativo: la percepción del profesorado en formación. Revista Electrónica Interuniversitaria de Formación del Profesorado, 18(3), 99-112. doi: http://dx.doi.org/10.6018/reifop.18.3.199321

Grant, B. K., \& Ray, J.A. (2013). Home, Shool, and Community Collaboration. London: Sage.

Lines, C., Milles, G. B., \& Arthur-Stanley, A. (2011). The Power of Family-school Partnering (FSP): A Practical Guide for School Mental Health Professionals and Educators. New York: Routledge/Taylor \& Francis Group.

Llevot, N. y Bernad, O. (2015). La participación de las familias en la escuela: Factores clave. Revista de la Asociación de Sociología de la Educación, 8(1), 57-70.

Machen, S., Wilson, J., y Notar, C. (2005). Parental involvement in the classroom. Journal of Instructional Psychology, 32(1), 13-16.

Márquez, L. (2014) La participación social en la escuela secundaria pública. Los padres y madres de familia como actores sociales: un estudio de caso (Tesis doctoral). Universidad Iberoamericana de Puebla, México.

Martínez, C. (2012). El muestreo en investigación cualitativa. Principios básicos y algunas controversias. Ciência \& Saúde Coletiva, 17(3), 613-619. Recuperado de https://www.scielosp.org/pdf/csc/2012.v17n3/613-619/es

Martínez, J. (1988). El estudio de casos en la investigación educativa. Investigación en la escuela, 6 , 41-50.

Mays, N. B. \& Pope, C. (2000). Qualitative research in health care: assessing quality in qualitative research. The BMJ Clinical Research, 320(7226), 50-52.

Montero, J. (2007). La fenomenología de la conciencia en E. Husserl. Universitas Philosophica, 24(48), 127-147.

Moratto, N. S., \& Zapata, J. J. y Messager, T. (2015). Conceptualización de ciclo vital familiar: una mirada a la producción durante el periodo comprendido entre los años 2002 a 2015. Revista CES Psicología, 8(2), 103-121.

Ñaupas, H., Mejía, E., Novoa, E., Villagómez, A. (2014). Metodología de la investigación cuantitativa-cualitativa y redacción de la tesis. Colombia: Ediciones de la U.

Patton, M. Q. (2002). Qualitative research and evaluation methods. Thousand Oaks: Sage.

Perales, F. J., y Escobedo, M. M. (2016). La participación social en la educación: entre propuestas innovadoras u tradición educativa. Revista Electrónica de Investigación Educativa, 18(1), 69. 81. Recuperado de http://redie.uabc.mx/redie/article/view/738

Reeder, H. P. (2011). La praxis fenomenológica de Husserl. Bogotá, Colombia: San Pablo.

Salgado, A. C. (2007). Investigación cualitativa: diseños, evaluación del rigor metodológico y retos, Liberabit, 13(13), 71-78.

Trujillo, F. (2012). Propuestas para una escuela en el siglo XXI. Madrid: Los Libros de la Catarata.

Valdés, Á. A., Martín, M. y Sánchez, P. A. (2009). Participación de los padres de alumnos de educación primaria en las actividades académicas de sus hijos. Revista Electrónica de Investigación Educativa, 11 (1), 1-17. Recuperado de: https:/www.redalyc.org/pdf/155/15511137012.pdf 